\title{
HAS INCREASED BODY WEIGHT MADE DRIVING SAFER? ${ }^{\dagger}$
}

\author{
RICHARD A. DUNN ${ }^{\mathrm{a}, *}$ and NATHAN W. TEFFT ${ }^{\mathrm{b}}$ \\ ${ }^{a}$ Texas A\&M University, TX, USA \\ ${ }^{\mathrm{b}}$ University of Washington, WA, USA
}

\begin{abstract}
We develop a model of alcohol consumption that incorporates the negative biological relationship between body mass and inebriation conditional on total alcohol consumption. Our model predicts that the elasticity of inebriation with respect to weight is equal to the own-price elasticity of alcohol, consistent with body mass increasing the effective price of inebriation. Given that alcohol is generally considered price inelastic, this result implies that as individuals gain weight, they consume more alcohol but become less inebriated. We test this prediction and find that driver blood alcohol content (BAC) is negatively associated with driver weight. In fatal accidents with driver BAC above 0.10 , the driver was 7.8 percentage points less likely to be obese than drivers in fatal accidents that did not involve alcohol. This relationship is not explained by driver attributes (age and sex), driver behaviors (speed and seatbelt use), vehicle attributes (weight class, model year, and number of occupants), or accident context (county of accident, time of day, and day of week). Copyright (C) 2013 John Wiley \& Sons, Ltd.
\end{abstract}

Received 29 November 2012; Revised 19 July 2013; Accepted 25 July 2013

JEL Classification: $\mathrm{H} 23 ; 118 ; \mathrm{K} 42$

KEY WORDS: obesity; drunk driving; alcohol consumption

\section{INTRODUCTION}

The biological relationship between weight and blood alcohol content (BAC), the primary determinant of impairment from alcohol consumption, is well-known. Conditional on the amount of alcohol consumed, BAC is negatively related to the amount of water in the body, which tends to increase with body mass. Widmark (1932) first proposed a method for calculating BAC, today known as the 'Widmark formula'

$$
B=\frac{A}{r W}-\beta t
$$

where $B$ is BAC, $A$ is the mass of alcohol consumed, $r$ is the Widmark factor, $W$ is the body weight, $t$ is the time elapsed since imbibing commenced, and $\beta$ is the elimination rate. ${ }^{1}$ All else constant, individuals with greater body mass will tend to exhibit a lower BAC and as a result are less impaired. ${ }^{2}$

\footnotetext{
*Correspondence to: Texas A\&M University, TX, USA. E-mail: dunn.econ@gmail.com

${ }^{1}$ The Widmark factor is typically estimated according to characteristics that include percentage body fat, age, and sex (Posey and Mozayani, 2007). For example, the Widmark factor is frequently estimated to be larger for males.

${ }^{2}$ The effect of body mass on BAC is illustrated in alcohol impairment charts commonly produced by law enforcement agencies to caution potential drivers about the consequences of driving after consuming alcohol. They list approximate BAC based on the number of drinks consumed, the time elapsed since consumption began, weight, and gender. See, for example, the charts published by the University of Oklahoma Police Department: http://www.ou.edu/oupd/bac.htm (last accessed October 23, 2012)

${ }^{\dagger}$ Supporting information may be found in the online version of this article
} 
Although body mass and BAC have a negative biological relationship, if individuals with higher body masses change their total alcohol consumption in response to the reduced marginal effect of each drink, the observed relationship between weight and inebriation would differ from the biological relationship. Therefore, this study considers how body mass mediates the relationship between alcohol consumption and inebriation and thus the risk of a fatal motor vehicle accident.

First, we develop a utility maximizing model of alcohol consumption that incorporates the biological relationship between alcohol consumption, weight, and inebriation. The model yields two important equilibrium conditions as follows: (i) the elasticity of alcohol demand with respect to weight is equal to one plus the own-price elasticity of alcohol; (ii) the elasticity of inebriation with respect to weight is equal to the own-price elasticity of alcohol. That the elasticities of alcohol consumption and inebriation with respect to weight are both related to the own-price elasticity of demand reflects the fact that higher body mass effectively raises the price of inebriation. As demand analyses typically estimate alcohol to be inelastic (Gallet, 2007), the model predicts that weight gain is positively related to alcohol consumption but negatively related to the chosen inebriation level. Although the former is well-documented (French et al., 2010), to our knowledge, the latter remains unidentified, and its implications for public health outcomes remain unexplored.

Although it is relatively straightforward to examine the relationship between weight and alcohol consumption, heterogeneity in the Widmark factor, $r$ in equation (1), makes it difficult to directly assess BAC from datasets that include self-reported alcohol consumption. One source of measured BAC, however, is from drivers involved in alcohol-related motor vehicle accidents, and a testable implication of our model is that conditional on other risk factors, obese drivers should be less likely to be involved in alcohol-related motor vehicle accidents. In addition, the negative consequences of alcohol consumption are of greater public policy concern than inebriation in and of itself. Therefore, we explore the relationship between weight and inebriation using information on automobile accidents involving only one vehicle between 2000 and 2009 from the Fatality Analysis Reported System (FARS).

In fatal accidents involving only one vehicle, we find that deceased drivers with non-zero BAC levels weigh significantly less than deceased drivers who showed no alcohol intoxication. The obesity rate among the former is $13.6 \%$ compared with $20.8 \%$ in the latter. Regression analysis that includes all confounding risk factors in FARS reveals that this relationship is not explained by differences in driver attributes (e.g., age and gender), driver behaviors (e.g., speed and seatbelt use), vehicle attributes (e.g., weight class, model year, and number of occupants), or accident context (e.g., county of accident, time of day, day of week, weather, and distance from residence).

These findings are consistent with the theoretical predictions of our model, and alternative explanations are either rejected by the existing literature, for example, a cushion effect of obesity or not supported by auxiliary analyses of FARS data, for example, obese drivers choosing to drink closer to home. This leads us to conclude that the physiological relationship between body mass and impairment influences the equilibrium level of inebriation chosen by consumers and thus the risk of a fatal motor vehicle accident.

\section{OBESITY, ALCOHOL CONSUMPTION, AND DRIVING BEHAVIOR}

Although we are unaware of previous literature directly exploring the relationship between weight and the likelihood of involvement in drunk driving accidents, there are a number of related studies investigating obesity and driving behavior, which highlight the importance of controlling for measures of risky driving behavior in the empirical analysis. Anderson et al. (2012) find that commercial motor vehicle operators with higher BMI while training for their commercial driver's licenses were more likely to be in a subsequent accident. Simmons and Zlatoper (2010) estimate that during 2005, accident fatalities per mile traveled was positively associated with a state's obesity prevalence.

Rashad et al. (2006) find mixed evidence for the relationship between gasoline taxes and weight, suggesting that although higher taxes might encourage more walking, they could also reduce mobility to purchase healthy food. Courtemanche (2010) finds a negative relationship between gasoline prices and weight, hypothesizing mechanisms 
including more walking and less restaurant dining when prices are high. Other studies find a relationship between urban sprawl or the built environment and obesity, with differences in driving behavior one likely explanation (Zhao and Kaestner, 2010). Li et al. (2011) consider passenger vehicle sales and report that counties with higher obesity rates also exhibit stronger demand for larger and less fuel efficient vehicles. Anderson and Aufhammer (2011) quantify the fatality externality of being hit by a heavier vehicle in a collision. Related research has identified a positive effect of obesity on gasoline consumption (Jacobson and McLay, 2006; Jacobson and King, 2009).

There are important biological and physiological, in addition, or economic or social, mechanisms at play when considering how obesity relates to drunk driving accidents. Because Widmark (1932) first quantified the association between BAC and weight, other researchers have advanced knowledge of the physiological relationship while reinforcing body weight as an important determinant of BAC. Posey and Mozayani (2007) summarize recent models for predicting BAC for use by toxicology practitioners. The legal criterion for drunk driving refers specifically to a BAC threshold has support. For example, in an experimental setting, Moskowitz et al. (2000) tested an impairment using a driving simulator and a divided attention task, finding that performance was impaired as BAC increased to 0.1.

This paper also contributes to the small but growing literature in economics describing the interaction between health-related behaviors (other than alcohol consumption) and motor vehicle accidents. Chaloupka and Laixuthai (1997) find that any reduction in the price of marijuana (for example, through reduced legal sanctions) would lead youths to substitute away from alcohol and reduce drunk driving, which would more than offset the negative consequences of 'stoned driving'. Adams and Cotti (2008) estimate that drunk driving accidents increase after bar smoking bans are implemented in areas bordering jurisdictions without bans. Cotti and Walker (2010) estimate that, particularly in rural areas, the number of drunk driving accidents increases in counties nearby where a casino opens. Freeman and McManus (2010) find that opening an additional substance abuse treatment clinic in a small US county reduces drunk driving fatalities by $15 \%$. Jackson and Owens (2011) investigate the relationship between public transportation and criminal behavior associated with drinking, and they find that bars within walking distance of public transportation are associated with higher rates of alcohol-related arrests but lower levels of drunk driving arrests.

\section{A MODEL OF ALCOHOL CONSUMPTION AND WEIGHT}

Consider an individual who derives utility from two sources as follows: inebriation level $q$ and the numeraire composite good $x$. Further, inebriation is an increasing function of alcohol consumption $A$, which is sold at price $p$, and decreasing in weight $W$. Although inebriation directly increases utility, it also increases the probability of a negative utility shock such as an automobile accident, determined by the mean function $f$. Given income level $m$, the expected utility maximization problem is

$$
\begin{aligned}
& \max _{A, x} U(q, x)-f(q), \text { s.t. } \\
& q=q(A, W) \\
& p A+x \leq m
\end{aligned}
$$

Assuming $U$ and $f$ are twice continuously differentiable and that inebriation level $q$ is determined by the Widmark equation (equation 1), the first-order conditions imply

$$
\frac{1}{W r}\left(U_{q}-f^{\prime}\right)=p U_{x}
$$

Intuitively, an additional unit of alcohol increases inebriation by $1 / \mathrm{Wr}$, and inebriation increases net utility by $U_{q}-f^{\prime}$. Instead of purchasing one unit of alcohol, one could purchase $p$ units of the composite good, each with marginal utility $U_{x}$. 
Totally differentiating the first-order condition with respect to $A$ and $W$ reveals the relationship between the elasticity of alcohol consumption with respect to weight and the own-price elasticity of alcohol (see Appendix A)

$$
\varepsilon_{A, W}=\varepsilon_{A, p}+1
$$

Applying a log approximation to a static version of the Widmark equation that ignores the diminution of BAC over time yields

$$
\% \Delta B=\% \Delta A-\% \Delta W,
$$

so that a $1 \%$ increase in weight decreases BAC by $\varepsilon_{A, W}-1$. Substituting this into (3) shows that the elasticity of inebriation with respect to weight is equal to the own-price elasticity of alcohol

$$
\varepsilon_{B, W}=\varepsilon_{A, p}
$$

That the elasticities of alcohol consumption and inebriation with respect to weight are both related to the own-price elasticity of demand reflects the fact that higher body mass effectively raises the price of inebriation. As demand analyses typically estimate alcohol to be inelastic (Gallet, 2007), the results in (3) and (4) imply that weight gain increases total alcohol consumption while decreasing the chosen inebriation level.

It is worth pointing out that the price elasticity of alcohol need not be constant across all individuals or income levels, and thus the elasticity of inebriation with respect to weight need not be constant. For example, if as expected, the price responsiveness of alcohol varied negatively with income level, that is, individuals with lower income exhibited more elastic demand, we would also expect that the inebriation level of individuals with lower income to be more weight responsive. As obesity is negatively associated with income level, a corollary is that the inebriation level of individuals with higher body mass will also be more weight responsive.

\section{EMPIRICAL APPROACH}

The model developed in the preceding section predicts that because alcohol is price inelastic, inebriation level is decreasing in weight. Where BAC level is observable, one could directly test this prediction by studying the relationship between chosen BAC and weight either across individuals (cross-sectionally) or across time within individual (longitudinally). However, unobserved heterogeneity in the effect of alcohol consumption on inebriation renders calculation of BAC from self-reports of alcohol consumption infeasible.

One reliable source of BAC data comes from police reports of motor vehicle accidents. Thus, instead of directly examining whether individuals select lower inebriation levels as their body mass increases, we utilize the model to generate testable implications about the joint distribution of BAC and weight among drivers involved in fatal motor vehicle accidents. Define the mean weight of drivers who are involved in a one-car fatal accident as

$$
W_{A}=\int_{x} \int_{b} x p(A \mid x, b) f(x, b)
$$

where $x$ denotes the weight of the driver, $b$ denotes the BAC of the driver, $p(\mathrm{~A} \mid x, b)$ is the probability that a driver of weight $x$ and BAC $b$ is involved in an accident, and $f(x, b)$ is the joint distribution of the driver's weight and BAC. Differences in the mean weights across driver types can be explained either by differences in the risk profile of drivers, $p(\mathrm{Al} x, b)$, or the weight distribution of individuals at different BAC levels, $f(x, b)$. If equilibrium inebriation decreases in weight, then conditional on the driver's risk, the mean weight of drivers in fatal accidents should be decreasing in BAC.

Intuitively, if obese individuals select a lower inebriation level, a randomly selected driver with a high BAC level would weigh less (in expectation) than a randomly selected driver with a low BAC level once we have 
conditioned on differences of the driver's risk profile. We therefore propose the following reduced-form regression analysis using the data on driver fatalities in one-car accidents

$$
y_{i c t}=\beta_{0}+a_{i c t} \beta_{a}+x_{i c t} \beta_{x}+z_{i c t} \beta_{z}+\lambda_{t}+\delta_{c}+\varepsilon_{i c t}
$$

where $y$ is a measure of driver weight (BMI and obesity status); $a$ is the level of driver drunkenness measured by BAC; $x$ is a vector of driver demographics and characteristics intended to measure the riskiness of driving behavior, for example, age, gender, seatbelt use, driving speed, distance driven, vehicle type, vehicle weight, time of day, and location of accident, as well as a vector $z$ of contextual variables describing the driver's state of residence, including the state's unemployment rate, gasoline tax, per capita beer consumption, beer tax, and cigarette tax, and finally, all models include a vector of time-varying indicator variables for year, quarter, day of week, and hour, as well as a location-varying vector of indicator variables for the county in which the accident occurred. The standard errors reported for the regression models are heteroskedasticity-robust and clustered at the county level.

As an alternative to explore how the expected body mass of drivers vary with their BAC level, one could reverse the analysis and consider how the expected BAC of drivers vary with their body mass, that is, replace BAC as the dependent variable and include body mass as an independent variable. Because we can only observe the BAC of drivers involved in fatal accidents, neither this specification nor equation (6) provide any structural identification of our theoretical model but are rather alternative ways of testing the same prediction about the joint distribution of body mass and inebriation level. To us, it seems more intuitive to study the mean weight of drivers conditional on differences in risk profile, but we recognize that this is largely a matter of taste. For the interested reader, in Appendix C, we also report coefficient estimates from a series of linear regressions of driver BAC on measures of body mass (Table $\mathrm{C} 1$ ), a series of conditional logit regressions of BAC interval on body mass (Table C2), and a series of quantile regressions of BAC on body mass (Table C3). ${ }^{3}$

Equation (6) allows us to study whether conditional on being in a fatal accident and controlling for an exhaustive list of explanatory variables associated with risk preference and driving behavior, the mean weight of drivers is higher or lower when driver BAC is higher. If the mean weight of drivers is decreasing in impairment level because of the biological relationship between alcohol consumption and inebriation, then we would expect the coefficient estimate on $a$ to be negative. Of course, there are other plausible mechanisms that could also generate a negative relationship between BAC and BMI. For example, this could arise if obese drivers tended to drink less than non-obese drivers, but previous empirical studies reject this hypothesis (French et al., 2010).

Alternatively, obese individuals may exhibit higher survival rates conditional on being in a motor vehicle accident. Although it might be expected that obese persons would benefit from a 'cushion effect' (Arbabi et al., 2003) in car accidents, the existing literature addressing this hypothesis yields ambiguous and/or conflicting results (Arbabi et al., 2003; Kent et al., 2010; Sivak et al., 2010; Ma et al., 2011). However, the most recent research using the largest sample of accidents and the most comprehensive controls for confounding factors found that slightly obese drivers (BMI between 30 and 35 years) were no more likely to survive a motor vehicle accident compared with normal weight drivers and more obese drivers (BMI $>35$ years) were less likely to survive a motor vehicle accident (Jehle et al., 2012). A second study using similar information from driver's licenses finds that driver obesity increases fatality risk in a motor vehicle accident (Rice and Zhu, 2013). Thus, there appears to be little evidence that differences in mortality conditional on being in an accident would explain a negative relationship between driver BMI and BAC, and the best evidence suggests that differences in mortality risk will bias our estimate toward zero.

\footnotetext{
${ }^{3}$ It is important to recognize that using aggregate data to overcome the lack of observable BAC is not generally a valid empirical approach. From a practical standpoint, detrended changes in mean state BMI or state obesity rates are subject to enormous measurement error. From an identification standpoint, it clearly matters who has gained weight and thus whose selected inebriation level may have changed. Relating a single moment of the BMI distribution to state-level rates of drunk driving fatalities is not sufficient to identify the relationship implied by the theoretical model.
} 
Finally, obese drinkers may choose to mitigate their risk of being involved in a fatal accident for reasons unobserved to the econometrician. For example, FARS does not include information on driver income, marital status, or parental status, which are attributes that could affect the driving decisions of those who drink. The most direct form of risk mitigation is decreasing the distance driven while intoxicated, which, at its limit, is manifested as choosing not to drink and drive at all. Because of the nature of FARS data-only information on drivers in fatal accidents is available-we cannot investigate the attributes of those who choose not to drive. We can, however, explore the distance traveled to consume alcohol among those involved in accidents. If obese drivers were choosing to drink closer to home, then we would expect to see that accidents involving obese drivers were more likely to occur near their residences. Therefore, the issue of where accidents take place relative to the residence of the driver will be explored in our robustness checks. We find that distance between accident location and residence is very weak and positively related to obesity status, and thus there is no evidence that obese drivers are decreasing their relative risk of accident involvement by driving shorter distances while intoxicated. This gives us confidence that obese drivers are not also differentially deciding not to drink and drive at all.

\section{DATA}

To explore how obesity mediates the relationship between alcohol consumption and inebriation, and thus risk of a fatal motor vehicle accident, we use the National Highway Traffic Safety Administration's (NHTSA) FARS, which provides information on all fatal car accidents in the USA. We restrict attention to accidents that involved only one vehicle in order to be able to clearly identify the relationship between weight and alcohol consumption of the driver who made the error leading to the accident (at least, if any driver was at fault). Because this is the standard data source for studying accident fatalities, we focus the discussion here on the most important aspect of the FARS with respect to the current study: the height and weight of drivers in fatal accidents.

Starting in 1998, FARS reports height and weight information from driver's licenses (the sample analyzed subsequently includes only the decade between 2000 and 2009 after merging with state tax rates). Driver BMI is then calculated as reported weight (in kilograms) divided by the square of reported height (in square meter). Biologically implausible values (of less than 13 or greater than 55) are dropped. ${ }^{4}$

Three issues arise with using self-reported height and weight from driver's licenses. First, systematic misreporting of height and weight in survey data is well-documented. Individuals tend to over-report their height and underreport their weight, resulting in calculated BMI that is lower than the true value. Second, information on a driver's license may be several years old. Although height shows little variation over short time spans, this is not true of weight. At the least, this generates additional noise in our BMI measure. More problematic, however, is that the distribution of weight in the US population exhibits a distinct hump-shaped age profile peaking between 65 and 70 years. If weight initially increases with age, using a weight measure from an earlier period would tend to further exacerbate the underreporting of weight among younger drivers, although it may mitigate this problem among older drivers for whom weight tends to decrease with age. Third, seven relatively populous states (Connecticut, Florida, Massachusetts, New Jersey, New York, North Carolina, and Pennsylvania) do not list weight on driver's licenses, reducing the number of observations available for estimation.

Despite these issues, we argue that self-reported height and weight from driver's licenses are sufficient to study the problem at hand. First, validation studies of height and weight information from driver's licenses show a high correlation between reported and measured values (Le Marchand et al., 1988; Ossiander et al., 2004). Although there is undoubtedly measurement error in data collected from driver's licenses, the underlying signal

\footnotetext{
${ }^{4}$ These values are criteria generally used by the Centers for Disease Control (CDC). See, for example, http://www.cdc.gov/healthyyouth/ YRBS/pdf/national_usersmanual_yrbs.pdf (last accessed on October 23, 2012).
} 
Table I. Impairment levels of drivers in one-car fatal accidents

\begin{tabular}{lcr}
\hline Unconditional mean BAC & Proportion of drivers with \\
$0<$ BAC $<0.1$ & $\begin{array}{c}\text { Proportion of drivers with } \\
\text { BAC } \geq 0.1\end{array}$ \\
\hline $0.0883(0.0006)$ & $0.0928(0.0017)$ & $0.4215(0.0028)$ \\
\hline
\end{tabular}

BAC, blood alcohol content.

The sample includes FARS data from 2000 to 2009. Standard errors are in parentheses. $N=30,203$.

to noise ratio is still relatively high. Assuming that the magnitude of this error is not systematically related to propensity for drunk driving (we are aware of no evidence to suggest this), FARS height and weight data should not perform appreciably worse in regression analysis than self-reported data from surveys. In studies that have compared results using self-reported information with measurements collected during a clinical exam, researchers tend to find that misreporting has no meaningful effect on estimation, for example, Cawley (1999). Instead, the tendency to underreport weight is simply absorbed by the constant term. Hence, there are reasonable a priori arguments that measurement error should not influence the results reported subsequently.

Second, the reporting instructions for FARS indicate that data on driver weight and height should be taken from the driver's license, but 'The Coroner's Report may be used and may contain more current/accurate information' (NHTSA, 2010, page 375). Thus, there are records in FARS for which measured height and weight are available. For most states, this is of limited value because the source of height and weight information (driver's license or coroner's report) is not recorded. However, for the seven states that do not provide weight information on their driver's license, the coroner's report is the only possible source for this information. Hence, as another robustness check, we repeat the analysis using only accidents with reported weight involving driver's licenses issued in these seven states (implying that weight was recorded by the coroner). The results are similar to those using the full FARS sample, giving us confidence that measurement issues with self-reported height and weight are not driving the results reported subsequently. ${ }^{5}$

The BAC of drivers involved in fatal accidents is of primary interest, but for approximately $60 \%$ of accidents BAC is missing because an alcohol test is either not administered or the result is not reported (NHTSA, 2002a). Because BAC is the primary independent variable of interest, we use only observations with non-missing values for the full analysis. To provide evidence that selection into non-missing BAC is not biasing the results, however, we repeat the main analysis employing multiple imputation methods specifically developed by NHTSA for use with the FARS data when driver BAC was not recorded in the original accident report. ${ }^{6}$ These results are reported in Table B2 in the Appendix, and it is evident that imputation has no meaningful effect on the coefficient estimates of interest.

We define categories of driver BAC in order to separate accidents according to the risk posed by alcohol in each case. A non-alcohol-related fatal accident is defined as an accident in which the driver is identified as having a BAC value (assigned or imputed) of 0 , and the categories low alcohol-related fatal accident (LARFA) and high alcohol-related fatal accident (HARFA) are defined similarly but with non-zero BAC values less than 0.10 and greater than or equal to 0.10 , respectively. Within the sample of one-car fatal accidents, the unconditional mean BAC of drivers is 0.088 , just over the legal per se definition of driving under the influence (Table I). Over $40 \%$ of drivers involved in a one-car fatal accident exhibit some level of alcohol impairment, and about one-third have a BAC above 0.1 . That such a large proportion of fatal accidents involve at least some alcohol clearly illustrate the increased risk that comes with driving under its influence.

\footnotetext{
${ }^{5}$ The sample of driver's licenses eligible for conducting this robustness check is small $(N=254)$ so the coefficients of interest in models including the full set of controls do not reach statistical significance. Nevertheless, it is reassuring that the signs of the coefficients remain the same as reported in the main findings across a range of specifications including various controls, and in some cases, the coefficients reach statistical significance.

${ }^{6}$ Details of the imputation method are outlined in NHTSA (2002b). Reported estimates and standard errors involving an imputed value (i.e., $\mathrm{BAC}$ or any variables derived from BAC) incorporate imputed values using the 'mi' suite of commands in STATA (version 11.2).
} 
Table II. Summary statistics, one-car accidents

\begin{tabular}{|c|c|c|c|c|}
\hline & Mean & Standard deviation & Min. & Max. \\
\hline Driver BMI & 25.73 & 5.06 & 13.43 & 54.39 \\
\hline Driver obese & 0.171 & 0.377 & 0 & 1 \\
\hline Driver age & 36.4 & 16.6 & 7 & 99 \\
\hline Driver male & 0.765 & 0.424 & 0 & 1 \\
\hline Occupants & 1.638 & 1.104 & 1 & 20 \\
\hline Vehicle model year & 1996.3 & 6.5 & 1966 & 2010 \\
\hline Driver used restraint & 0.395 & 0.489 & 0 & 1 \\
\hline Travel speed up to $97 \mathrm{mph}$ & 65.0 & 20.1 & 0 & 97 \\
\hline Travel speed $\geq 97 \mathrm{mph}$ & 0.143 & 0.350 & 0 & 1 \\
\hline Previous crashes & 0.204 & 0.514 & 0 & 10 \\
\hline Previous suspensions or revocations & 0.422 & 1.228 & 0 & 27 \\
\hline Previous DWI convictions & 0.078 & 0.327 & 0 & 7 \\
\hline Previous speeding convictions & 0.363 & 0.772 & 0 & 11 \\
\hline Previous other harfmul MV convictions & 0.327 & 0.806 & 0 & 15 \\
\hline Any previous crashes & 0.165 & 0.372 & 0 & 1 \\
\hline Any previous suspensions or revocations & 0.198 & 0.398 & 0 & 1 \\
\hline Any previous DWI convictions & 0.064 & 0.245 & 0 & 1 \\
\hline Any previous speeding convictions & 0.243 & 0.429 & 0 & 1 \\
\hline Any previous other harmful MV convictions & 0.210 & 0.408 & 0 & 1 \\
\hline Unemployment rate & 5.808 & 2.075 & 2.3 & 13.4 \\
\hline Gasoline tax & 0.213 & 0.063 & 0.0021 & 0.455 \\
\hline Beer consumption, per capita & 22.83 & 3.35 & 12.1 & 32.2 \\
\hline Beer tax & 0.254 & 0.213 & 0 & 1.07 \\
\hline Cigarette tax & 0.600 & 0.494 & 0.0111 & 3.46 \\
\hline Peak drinking hours & 0.238 & 0.426 & 0 & 1 \\
\hline
\end{tabular}

DWI, driving while intoxicated; MV, moving violation.

The sample includes FARS data from 2000 to 2009. Not reported here (but included in the regression models) are 13 car and truck weight classes.

$N=30,203$.

Table II reports the summary statistics for the sample of one-car accidents used in the regression analysis. After excluding missing values for the covariates, the remaining sample consists of 30,203 accidents. The table reports that drivers in one-car accidents were approximately 36 years old, on average, and the percent of drivers who were male is $77 \%$. Also reported are measures intended to proxy for the riskiness of driving behavior, including whether or not the driver wore a restraint device and the speed the driver was traveling when the accident occurred. A collection of counts and indicators of whether or not the driver had any previous events hypothesized to be correlated with risky driving are also summarized in the table. These include driver's license suspensions or revocations, crashes, driving while intoxicated (DWI) convictions, speeding convictions, and any previous other harmful moving violation (MV) convictions.

Drawn from the Local Area Unemployment Statistics of the Bureau of Labor Statistics, US Department of Labor, the average state unemployment rate for drivers in the sample is reported. ${ }^{7}$ The per gallon state gasoline tax, per gallon state beer tax, and per pack state cigarette tax are collected from the Tax Foundation, ${ }^{8}$ and per capita annual beer consumption (in gallons) is collected from the Brewer's Almanac produced by the Beer Institute. ${ }^{9}$ All taxes are reported in dollars. Peak drinking hours are defined as those hours between 8 PM and $4 \mathrm{AM}$ on Friday and Saturday nights, and the table shows that almost one quarter of all fatal accidents occur during peak drinking hours.

\footnotetext{
${ }^{7}$ http://www.bls.gov/lau/home.htm (last accessed October 23, 2012)

${ }^{8} \mathrm{http}: / /$ taxfoundation.org/ (last accessed October 23, 2012)

${ }^{9} \mathrm{http} / / / \mathrm{www}$. beerinstitute.org/ (last accessed October 23, 2012)
} 


\section{RESULTS}

The first column of Table III reports coefficient estimates of driver BMI on driver characteristics from our sample of fatal single-car accidents in the USA between 2000 and 2009 when controlling for potential differences in driver risk behavior. All regressions include indicator variables for vehicle weight class, county, year, quarter, day of week, and hour and use the imputed values of BAC provided by NHTSA (only the coefficient estimates on the indicator variables for driver BAC are presented; full estimation results are found in the Appendix Table B1). The BMI of drivers who died in fatal car accidents was significantly lower when alcohol was involved, even after controlling for measures of risky driving. For example, drivers with BAC above 0.10 tended to have a BMI that was 0.97 points lower than drivers with BAC of 0.0 (results when a continuous measure of BAC is used as the explanatory variable are considered subsequently).

The second column reports coefficients from an analogous linear probability model when driver BMI is replaced with obesity status. The probability that the driver in a fatal one-car accident was obese is decreasing with the BAC of the driver. Drivers in fatal accidents with BAC above zero but below 0.10 were 5.6 percentage points less likely to be obese. In fatal accidents with BAC above 0.10 , the driver was 7.8 percentage points less likely to be obese.

All regressions include controls for time of day, day of week, quarter, and year, implying that the results are not determined by differences in temporal patterns of driving between obese and non-obese individuals. To further test that this set of controls is sufficient, the third and fourth columns present results when we re-estimate the regressions (including all time controls) except restricting the sample to include only accidents that occurred during peak drinking hours, defined as during the hours from 8 PM to 4 AM on Friday and Saturday nights. Although the coefficients are somewhat lower in magnitude (in absolute value), they remain statistically significant and substantial with the same sign, particularly for HARFAs. The coefficients remain statistically significant in spite of the reduced statistical power when estimating this sample of 7185 accidents with a large number of covariates, including 1570 county indicator variables.

Before further considering the robustness of the LARFA and HARFA coefficients, it is worth discussing some of the estimated covariate coefficients (the full set of coefficient estimates are reported in Table B1 of the Appendix, which is available online). The signs of the coefficients for age and sex are broadly consistent with findings from previous obesity research (for example, see Chou et al., 2004). The number of occupants in the vehicle has a positive relationship with weight but not during peak drinking hours. When the estimates are significant (in only one model), drivers of newer vehicles are slightly heavier. Conditional on vehicle class weight (included as indicator variables), the driving behavior of heavier persons at first appears mixed. Greater weight is associated with a lower likelihood of wearing a seatbelt for the full sample. On the other hand, higher BMI is associated with fewer previous DWI or speeding convictions. But if the distribution of BAC is shifted to the left for higher levels of BMI, as we are suggesting, even if heavier drivers engage in riskier driving behaviors, all else equal, their likelihood of getting a DWI or speeding conviction may be reduced.

Table III. The association between driver weight and BAC categories

\begin{tabular}{lccrrr}
\hline & \multicolumn{2}{c}{ All hours $(N=30,203)$} & & \multicolumn{2}{c}{ Peak drinking hours $(N=7185)$} \\
\cline { 2 - 3 } & BMI & Obese & & BMI & Obese \\
\hline $0<$ Driver BAC $<0.1$ & $-0.8084^{* * *}(0.1007)$ & $-0.0556^{* * *}(0.0082)$ & & $-0.2146(0.2424)$ & $-0.0191(0.0190)$ \\
Diver BAC $\geq 0.1$ & $-0.9729^{* * *}(0.0740)$ & $-0.0776^{* * *}(0.0058)$ & & $-0.6358^{* * *}(0.2028)$ & $-0.0591^{* * *}(0.0148)$ \\
\hline
\end{tabular}

The first two columns present estimates using the full sample, whereas the last two columns present estimates using a sample restricted only to the hours from 8 PM to 4 AM on Friday and Saturday nights (defined as peak drinking hours). All regressions include the full set of accident and driver characteristics from FARS (see Table I) as well as indicator variables for vehicle weight class, county, year, quarter, day of week, and hour. *denotes statistically significant at $10 \%$ level. **denotes statistically significant at $5 \%$ level. ***denotes statistically significant at $1 \%$ level. 


\subsection{Robustness to specification}

Tables IV and V explore the robustness of our results to different specification choices. First, we consider the effect of using a continuous measure of inebriation (Table IV). When entering linearly, every 0.1 point increase in driver BAC is associated with $0.47 \mathrm{BMI}$ point increase in mean driver weight. Including a quadratic term reveals that very little concavity exists in the relationship. Increasing BAC from 0.0 to 0.2 only increases $\mathrm{BAC}^{2}$ by 0.04 , so that the large positive coefficient estimate belies an economically unimportant effect.

Although the literature generally finds a positive association between weight status and alcohol consumption, there is some evidence that very heavy drinkers tend to weigh less than non-drinkers (Lieber, 1991). In general, individuals who consume levels of alcohol sufficient to generate exceptionally high BAC levels may differ across a range of risk-taking characteristics that are not adequately controlled by our set of explanatory variables. To examine this possibility, Table $\mathrm{V}$ reports coefficient estimates when the analysis is repeated with an additional category to capture exceptional BAC levels. As we are just splitting an existing categorical variable, the coefficient estimate on the indicator of a positive BAC less than 0.1 is unaffected. Although drivers with BAC greater than 0.2 account for some of the negative association between driver weight and inebriation level, these cases are clearly not responsible for the entirety of the relationship. Therefore, we do not believe that individuals with low BMI and very high alcohol consumption are explaining our findings.

It is also worth noting that the regression results reported in Tables $\mathrm{C} 1-\mathrm{C} 3$ of the Appendix (available online) where the role of BAC and body mass are reversed, that is, the former is the dependent variable and the latter an independent variable, also reveal a negative association between the two.

Table IV. The association between driver weight and BAC

\begin{tabular}{|c|c|c|c|c|}
\hline & \multicolumn{4}{|c|}{ All hours $(N=30,203)$} \\
\hline & BMI & Obese & BMI & Obese \\
\hline \multirow[t]{2}{*}{$\begin{array}{l}\mathrm{BAC}^{2} \\
\mathrm{BAC}^{2}\end{array}$} & $-4.6543^{* * *}(0.3482)$ & $-0.3450 * * *(0.0251)$ & $\begin{array}{r}-5.6047 * * *(0.8922) \\
3.4348(3.1861)\end{array}$ & $\begin{array}{r}-0.4997 * * *(0.0649) \\
0.5590 * *(0.2211)\end{array}$ \\
\hline & \multicolumn{4}{|c|}{ Peak drinking hours $(N=7185)$} \\
\hline $\begin{array}{l}\text { BAC } \\
\text { BAC }^{2}\end{array}$ & $\begin{array}{c}\text { BMI } \\
-4.1524 * * *(0.8414)\end{array}$ & $\begin{array}{c}\text { Obese } \\
-0.3082 * * *(0.0624)\end{array}$ & $\begin{array}{c}\text { BMI } \\
-2.2389(1.7186) \\
-6.6067(4.7421)\end{array}$ & $\begin{array}{c}\text { Obese } \\
-0.3485^{* * * *}(0.1217) \\
0.1390(0.3143)\end{array}$ \\
\hline
\end{tabular}

BAC, blood alcohol content.

Each column represents a separate OLS model. The top panel presents estimates using the full sample, whereas the bottom panel presents estimates using a sample restricted only to the hours from 8 PM to 4 AM on Friday and Saturday nights (defined as peak drinking hours). All regressions include the full set of accident and driver characteristics from FARS (see Table I) as well as indicator variables for vehicle weight class, county, year, quarter, day of week, and hour. *denotes statistically significant at $10 \%$ level. **denotes statistically significant at $5 \%$ level. $* * *$ denotes statistically significant at $1 \%$ level.

Table V. The association between driver weight and BAC categories (with very high BAC)

\begin{tabular}{|c|c|c|c|c|}
\hline & \multicolumn{2}{|c|}{ All hours $(N=30,203)$} & \multicolumn{2}{|c|}{ Peak drinking hours $(N=7,185)$} \\
\hline & BMI & Obese & BMI & Obese \\
\hline $0<$ Driver $\mathrm{BAC}<0.1$ & $-0.8044 * * *(0.1007)$ & $-0.0554 * * *(0.0082)$ & $-0.2162(0.2426)$ & $-0.0193(0.0190)$ \\
\hline $0.1 \leq$ Driver $\mathrm{BAC}<0.2$ & $-0.7673 * * *(0.0797)$ & $-0.0662 * * *(0.0065)$ & $-0.5048^{* *}(0.2110)$ & $-0.0489 * * *(0.0157)$ \\
\hline Driver $\mathrm{BAC} \geq 0.2$ & $-1.2301 * * *(0.0950)$ & $-0.0919^{* * *}(0.0070)$ & $-0.8417 * * *(0.2278)$ & $-0.0751 * * *(0.0172)$ \\
\hline
\end{tabular}

BAC, blood alcohol content.

The first two columns present estimates using the full sample, whereas the last two columns present estimates using a sample restricted only to the hours from 8 PM to 4 AM on Friday and Saturday nights (defined as peak drinking hours). All regressions include the full set of accident and driver characteristics from FARS (see Table I) as well as indicator variables for vehicle weight class, county, year, quarter, day of week, and hour. *denotes statistically significant at $10 \%$ level. **denotes statistically significant at $5 \%$ level. ***denotes statistically sig nificant at $1 \%$ level. 
Table VI. The association between driver weight and accident distance from home

\begin{tabular}{|c|c|c|c|c|}
\hline & \multicolumn{2}{|c|}{ All hours $(N=32,339)$} & \multicolumn{2}{|c|}{ Peak drinking hours $(N=7,317)$} \\
\hline & BMI & Obese & BMI & Obese \\
\hline Distance (miles) & $0.0038(0.0026)$ & $0.0001(0.0002)$ & $0.0098 *(0.0057)$ & $0.0006(0.0005)$ \\
\hline
\end{tabular}

\subsection{Robustness to alternative explanations}

Although the preceding results are consistent with the theoretical prediction that individuals will choose a lower inebriation level as weight increases and thereby lower their risk of a fatal motor vehicle accident, they are also consistent with the alternative explanation that obese drivers are less likely to travel in order to consume alcohol. FARS includes geographic information on driver residence and accident site that enables us to construct a distance measure from the crash site to the driver's residence. ${ }^{10}$ Table VI reports regression coefficient estimates when replacing the LARFA and HARFA variables estimated in Table III with the calculated distance between accident and residence. There is no evidence that drivers with higher BMI drive shorter distances to consume alcohol.

\section{DISCUSSION AND CONCLUSIONS}

The preceding analysis demonstrates that obese drivers are less likely to be involved in an alcohol-related fatal accident. Moreover, the reduced risk associated with obese drivers is not fully accounted for by differences in driver characteristics or accident attributes. Although alternative plausible explanations exist that are also consistent with our findings, many of these are either rejected by the existing literature, for example, the cushion effect of obesity (Jehle et al., 2012), or are not supported by our auxiliary analyses, that is, obese drivers in fatal motor vehicle accidents travel as far as non-obese drivers to consume alcohol.

Yet, two issues with the empirical exercise are worth noting. First, we relied on information from drivers who had been involved in a fatal accident. This is obviously a highly selected sample. Although we include several explanatory variables that should be correlated with driver risk and/or time preference, for example, wearing a seatbelt and estimated speed, these are only proxies and may only partially account for heterogeneity across individuals. A high tolerance for risk and/or a strong preference for the present could lead to both more drinking and more obesity. In practice, this would work in the opposite direction of our results, so that our estimates of the relationship between BMI and BAC are conservative.

Second, although FARS includes extensively detailed information about accident and driver characteristics, two important demographic variables are missing: income or education. Empirical evidence suggests that higher income is associated with greater alcohol consumption (Gallet, 2007) but negatively related to obesity risk. Our controls for vehicle age and year may capture some of the differences in driver socio-economic status, but some bias may still remain.

\footnotetext{
${ }^{10}$ The distance is calculated using the spherical law of cosines formula (see http://www.movable-type.co.uk/scripts/latlong.html) for the distance between two coordinates on a sphere. The two sets of coordinates are the latitude and longitude of the accident and the latitude and longitude of the centroid of the zip code of the driver's residence. The accident coordinates and driver's zip code are recorded in FARS, and zip code centroids were obtained from a 1999 compilation produced by the Census Bureau (see http://databases.about.com/od/access/ a/zipcodedatabase.htm).
} 
Despite these issues, we believe that the evidence presented suggests that obesity itself, through the mitigating effect of greater body mass on alcohol impairment, reduces the risk of fatal drunk driving accidents. This is a potential benefit of obesity that accrues not only to the obese driver but also to passengers and other vehicle occupants and therefore may be a positive externality to obesity.

Under admittedly strong assumptions, we calculated a 'back-of-the-envelope' external benefit due to reduced drunk driving fatalities resulting from the increase in population weight at the end of the 20th and beginning of the 21 st centuries in the USA. The overall approach was to ask how many more external drunk driving fatalities (i.e., excluding a drunk driver's own death) would have occurred in 2009 had mean population BMI been set equal to that from an earlier year (we chose 1974 because it was the earliest available single-year estimate). The steps and results of this calculation are presented in Table VII.

The results suggest that the annual external benefit of reduced drunk driving fatalities due to increasing population weight in the USA was approximately $\$ 3.4 \mathrm{bn}$ in 2009. Expressed in per capita terms, the annual external benefit was $\$ 13.99$ per drive age person (age 16 years and above) in the United States in 2009, relative to the mean population weight in 1974 . This is about $10 \%$ of the estimated negative externality associated with insurance pooling in the private market (Bhattacharya and Bundorf, 2009) and roughly half of the $\$ 7 \mathrm{bn}$ in obesity-attributable prescription drug costs paid for by Medicare Part D in 2006 (Finkelstein et al., 2009). This is not an apples-to-apples comparison either, because we only considered the counterfactual mean BMI in 1974 instead of the counterfactual assigning all obese persons to non-obese status, in which case our estimate would likely have been higher.

This external benefit calculation should be taken with substantial caution, however, because of the several strong assumptions involved. It notably does not rely on results from the empirical estimation presented here, which was instead intended to demonstrate the feasibility and robustness of the relationship predicted by the theoretical model. This calculation does, however, rely on the assumption that the observed distribution of BAC among drivers in fatal accidents is equal to the distribution of BAC of all drivers on the road. It also relies on calculations assuming only mean, but not other relevant distributional, changes in BMI and BAC. Therefore, further research on the external benefit of obesity with respect to drunk driving that does not rely on these strict distributional assumptions is needed.

Table VII. Calculation of the annual alcohol-related motor vehicle accident external benefit due to rising obesity prevalence

Elasticity of BAC with respect to $\mathrm{BMI}^{\mathrm{a}}$

Adult population mean BMI in the USA, $1974^{\mathrm{b}}$

Adult population mean BMI in the USA, $2009^{\mathrm{b}}$

Percent increase in mean BMI between 1974 and 2009

Implied percent decrease in mean BAC

Mean BAC in alcohol-related accidents observed in $2009^{\mathrm{C}}$

Implied mean BAC in alcohol-related accidents in 2009 with 1974 mean BMI

Relative risk of causing an accident between BACs, 0.18 to 0.17 , for all ages 21 years and above

Assuming linear interpolation between BAC thresholds, implied risk increase for 1974 mean BMI

Fatalities in motor vehicle accidents involving alcohol in $2009^{\circ}$

Percent of alcohol-related fatalities that are external ${ }^{\mathrm{e}}$

Number of fatalities in 2009 that were external

Number of additional external fatalities that would have occurred in 2009 with 1974 mean BMI

Total external benefit implied by recommended value of statistical life saved in $2009^{\mathrm{f}}$ (\$millions)

Aggregate external benefit in the U.S. per mean BMI point (\$millions)

External benefit per person age 16 and over in the U.S. in 2009, relative to 1974 mean BMI $^{\mathrm{g}}$

$-0.51$

24.52

27.40

$11.73 \%$

$-5.98 \%$

0.170

0.181

1.27

$29.52 \%$

8974

$21.25 \%$

1907

563

$\$ 3,378$

$\$ 1,175$

$\$ 13.99$

${ }^{a}$ Derived from the theoretical model developed here, this is equal to the price elasticity of alcohol, here drawn from a meta-analysis (Wagenaar et al., 2009).

${ }^{\mathrm{b}}$ Weighted estimates calculated using data from the Integrated Health Interview Survey, with 1974 the earliest available year (Minnesota Population Center and State Health Access Data Assistance Center, 2012).

${ }^{\mathrm{c}}$ Includes imputed values.

${ }^{\mathrm{d}}$ See Table IV in Peck et al. (2008).

${ }^{\text {e}}$ Estimated using results from Levitt and Porter (2001).

${ }^{f}$ Using the Department of Transportation's recommended \$6 million value of a statistical life (Goldin and Merrick, 2011).

${ }^{\mathrm{g}}$ Intercensal population estimates available at http://www.census.gov/popest/data/intercensal/national/nat2010.html (last accessed May 15, 2013). 
It is important to emphasize that we do not argue in support of policies that encourage obesity in order to reduce the external costs of drunk driving. There is ample evidence that obesity and alcohol consumption in combination increases the risk of developing potentially fatal liver disease (Hart et al., 2010; Liu et al., 2010). Moreover, in light of the results identified by Simmons and Zlatoper (2010) and Anderson et al. (2012), indicating a positive association between obesity and motor vehicle fatalities, more research is needed. It is clear that the negative externality associated with drunk driving is appropriately addressed through aggressive government policy (Eisenberg, 2003; Dee et al., 2005; Grabowski and Morrisey, 2006).

Nevertheless, amidst an increasing focus on obesity, ${ }^{11}$ economic analysis plays an important role in identifying whether government action to reduce obesity is warranted on the basis of increasing overall economic efficiency. Although the potential sources of market inefficiency are well-known to economists (e.g., externalities, information asymmetries, and psychological or behavioral barriers to optimal decision-making), previous empirical studies that attempt to calculate the magnitude of the obesity externality yield relatively small estimates (Bhattacharya and Sood, 2007; Van Baal et al., 2008; Bhattacharya and Bundorf, 2009; Brunello et al., 2009). The foregoing results bring the attention of policymakers to another consideration when crafting anti-obesity policies. A full accounting of the external costs and benefits of obesity is necessary to judge whether market interventions aimed at altering its prevalence increase efficiency.

\section{APPENDICES}

The derivation of the inebriation elasticity with respect to weight is found in Appendix A. Both Appendix B, the full set of regression results, and Appendix $\mathrm{C}$, the regression results when BMI is the dependent variable and $\mathrm{BAC}$ is the independent variable, are available online.

\section{APPENDIX A: DERIVATION OF THE INEBRIATION ELASTICITY WITH RESPECT TO WEIGHT}

Consider an individual who gets utility from two sources, inebriation level, $q$, and the numeraire composite good, $x$. Further, inebriation is an increasing function of alcohol consumption, $A$, which is sold at price $p$ and decreasing in weight, $W$. Although inebriation directly increases utility, it also increases the probability of a negative utility shock determined by the mean function, $f$. Given income level $m$, the expected utility maximization problem is

$$
\begin{aligned}
& \max _{\mathrm{a}, \mathrm{x}} U(q, x)-f(q), \text { s.t. } \\
& q=q(A, W) \\
& p A+x \leq m
\end{aligned}
$$

Assuming $U, f$, and $q$ are twice continuously differentiable, the first-order conditions imply

$$
U_{q}-f^{\prime}=U_{x} W r p
$$

where we use the Widmark equation to proxy for inebriation and plug in for $\partial q / \partial A$. Totally differentiating the first-order condition with respect to $A$ yields

$$
\left(U_{q q}-f^{\prime \prime}+U_{q x} \frac{\partial x}{\partial q}\right)\left[\frac{\partial A}{\partial p} / W r\right]=W r p\left(U_{q x}+U_{x x} \frac{\partial x}{\partial q}\right)\left[\frac{\partial A}{\partial p} / W r\right]+U_{x} W r
$$

so that

\footnotetext{
${ }^{11}$ A search of the Pubmed database under the major topic of 'obesity' reveals 3730 articles published between 1999 and 2000 , compared with 11,996 articles published between 2009 and 2010.
} 


$$
\left[\frac{\partial A}{\partial p} / W r\right]=U_{x} W r /\left(U_{q q}-f^{\prime \prime}+U_{q x} \frac{\partial x}{\partial q}-W r p\left(U_{q x}+U_{x x} \frac{\partial x}{\partial q}\right)\right)=K W r
$$

Thus, the price derivative of alcohol consumption is

$$
\frac{\partial A}{\partial p}=K W^{2} r^{2}
$$

Totally differentiating the first-order condition with respect to $W$ yields

$$
\left(U_{q q}-f^{\prime \prime}+U_{q x} \frac{\partial x}{\partial q}\right)\left[\frac{\partial A / \partial W}{W r}-\frac{A}{W^{2} r}\right]=W r p\left(U_{q x}+U_{x x} \frac{\partial x}{\partial q}\right)\left[\frac{\partial A / \partial W}{W r}-\frac{A}{W^{2} r}\right]+U_{x} r p
$$

so that

$$
\left[\frac{\partial A / \partial W}{W r}-\frac{A}{W^{2} r}\right]=U_{x} r p /\left(U_{q q}-f^{\prime \prime}+U_{q x} \frac{\partial x}{\partial q}-W r p\left(U_{q x}+U_{x x} \frac{\partial x}{\partial q}\right)\right)=K r p
$$

Thus,

$$
\frac{\partial A}{\partial W}-\frac{A}{W}=K r^{2} W p=\frac{\partial A}{\partial p} \frac{p}{W}
$$

Multiplying both sides by W/A, yields

$$
\varepsilon_{A, W}-1=\varepsilon_{A, p}
$$

which is equivalent to (2) in the text.

\section{CONFLICT OF INTEREST}

Authors assert that this research has been conducted within the guidelines of ethical scientific practice, and they are not subject to a conflict of interest.

\section{ACKNOWLEDGEMENTS}

The authors would like to thank Chad Cotti, Jack Porter, and the conference participants at the American Society of Health Economics, International Health Economics Association, and Western Economics Association International meetings, particularly our discussants Irina Grafova, Charles Courtemanche, and Benjamin Hansen. We also thank seminar participants at Texas A\&M University and Sam Houston State University. This research was supported by the National Institutes of Health under Ruth L. Kirschstein National Research Service Award No. T32 MH18029-21 from the National Institute of Mental Health. All errors are the authors'.

\section{REFERENCES}

Adams S, Cotti CD. 2008. Drunk driving after the passage of smoking bans in bars. Journal of Public Economics 92: 1288-1305.

Anderson M, Aufhammer M. 2011. Pounds that kill: the external costs of vehicle weight. NBER Working Paper \# 17170. Anderson JE, Govada M, Steffen T, Thorne C, Varvarigou V, Kales S, Burks S. 2012. Health behavior and accident risk: obesity is associated with the future risk of heavy truck crashes among newly recruited commercial drivers. IZA Discussion Paper \# 6408.

Arbabi S, Wahl WL, Hemmila MR, Kohoyda-Inglis C, Taheri PA, Wang SC. 2003. The cushion effect. Journal of Trauma 54: 1090-1093.

van Baal P, Polder J, de Wit G, Hoogenveen R, Feenstra T, Boshuizen HC, Engelfriet P, Brouwer W. 2008. Lifetime medical costs of obesity: prevention no cure for increasing health expenditures. Public Library of Sciences Medicine 5: 242-249. 
Bhattacharya J, Bundorf MK. 2009. The incidence of the healthcare costs of obesity. Journal of Health Economics 28: 649-658.

Bhattacharya J, Sood N. 2007. Health insurance and the obesity externality. Advances in Health Economics and Health Services Research 17: 279-318.

Brunello G, Michaud PC, Sanz-de-Galdeano A. 2009. The rise of obesity in Europe: an economic perspective. Economic Policy 59: 551-584, 590-96.

Cawley J. 1999. Rational addiction, the consumption of calories, and body weight. Ph.D. dissertation, University of Chicago, Chicago, IL.

Chaloupka FJ, Laixuthai A. 1997. Do youths substitute alcohol and marijuana? some econometric evidence. Eastern Economic Journal 23: 253-276.

Chou S, Grossman M, Saffer H. 2004. An economic analysis of adult obesity: results from the Behavioral Risk Factor Surveillance System. Journal of Health Economics 23: 565-587.

Cotti CD, Walker DM. 2010. The impact of casinos on fatal alcohol-related traffic accidents in the United States. Journal of Health Economics 29: 788-796.

Courtemanche C. 2010. A silver lining? reconnection between gasoline prices and obesity. Economic Inquiry 49: 935-957.

Dee TS, Grabowsk DC, Morrisey MA. 2005. Graduated driver licensing and teen traffic fatalities. Journal of Health Economics 24: 571-589.

Eisenberg D. 2003. Evaluating the effectiveness of policies related to drunk driving. Journal of Policy Analysis and Management 22: 249-274.

Finkelstein EA, Trogdon JG, Cohen JW, Dietz W. 2009. Annual medical spending attributable to obesity: payer- and service-specific estimates. Health Affairs 28(5): w822-w831.

Freeman BA, McManus B. 2010. Substance abuse treatment and motor vehicle fatalities. Southern Economic Journal 76: 1032-1048.

French MT, Norton EC, Fang H, Maclean JC. 2010. Alcohol consumption and body weight. Health Economics 19: $814-832$.

Gallet CA. 2007. The demand for alcohol: a meta-analysis of elasticities. Australian Journal of Agricultural and Resource Economics 51: 121-135.

Goldin R, Merrick C. 2011. What's the value of a statistical life? STATS Articles 2011. Available from: http://stats.org/ stories/2011/value_statistical_life_jun27_11.html (last accessed July 6, 2011).

Grabowski DC, Morrisey MA. 2006. Do higher gasoline taxes save lives? Economics Letters 90: 51-55.

Hart CL, Morrison DS, Batty GD, Mitchell RJ, Smith GD. 2010. Effect of body mass index and alcohol consumption on liver disease: analysis of data from two prospective cohort studies. BMJ 340: c1240.

Jackson CK, Owens EG. 2011. One for the road: public transportation, alcohol consumption, and intoxicated driving. Journal of Public Economics 95: 106-121.

Jacobson S, King D. 2009. Measuring the potential for automobile fuel savings in the US: the impact of obesity. Transportation Research 14: 6-13.

Jacobson S, McLay L. 2006. The economic impact of obesity on automobile fuel consumption. The Engineering Economist 51: 307-323.

Jehle D, Gemme S, Jehle C. 2012. Influence of obesity on mortality of drivers in severe motor vehicle crashes. American Journal of Emergency Medicine 30: 191-195.

Kent RW, Forman JL, Bostrom O. 2010. A biomechanical investigation of crash injury mechanisms in the obese. Obesity 18: 749-753.

Le Marchand L, Yoshizawa CN, Nomura AM. 1988. Validation of body size information on driver's licenses. American Journal of Epidemiology 128: 874-877.

Levitt SD, Porter J. 2001. How dangerous are drunk drivers? Journal of Political Economy 109: 1198-1237.

Li S, Liu Y, Zhang J. 2011. Lose some, save some: obesity, automobile demand, and gasoline consumption. Journal of Environmental Economics and Management 61: 52-66.

Lieber CS. 1991. Perspectives: do alcohol calories count? American Journal of Clinical Nutrition 54: 976-982.

Liu B, Balkwill A, Reeves G, Beral V. 2010. Body mass index and risk of liver cirrhosis in middle aged UK women: prospective study. BMJ 340: c912.

Ma X, Laud PW, Pintar F, Kim J-E, Shih A, Shen W, Heymsfield SB, Allison DB, Zhu S. 2011. Obesity and non-fatal motor vehicle crash injuries: sex difference effects. International Journal of Obesity 35: 1216-1224.

Minnesota Population Center and State Health Access Data Assistance Center. 2012. Integrated Health Interview Series: Version 5.0. Minneapolis. Available from: http://ihis.us/.

Moskowitz H, Burns M, Fiorentino D, Smiley A, Zador P. 2000. Driver characteristics and impairment at various BACs. NATIONAL Highway Traffic Safety Administration, DOT HS 809 075, Washington, D.C.

NHTSA. 2002a. Estimates of alcohol involvement in fatal crashes. National Highway Traffic Safety Administration, DOT HS 809 450, Washington, D.C. 
NHTSA. 2002b. Transitioning to multiple imputation - a new method to impute missing blood alcohol concentration (BAC) values in FARS. National Highway Traffic Safety Administration, DOT HS 809 403, Washington, D.C.

NHTSA. 2010. Fatality Analysis Reporting System (FARS) coding and validation manual. National Highway Traffic Safety Administration, DOT HS 811 530, Washington, D.C.

Ossiander EM, Emanuel I, O'brien W, Malone K. 2004. Driver's licenses as a source of data on height and weight. Economics and Human Biology 2: 219-227.

Peck RC, Gebers MA, Voas RB, Romano E. 2008. The relationship between blood alcohol concentration (BAC), age, and crash risk. Journal of Safety Research 39: 311-319.

Posey D, Mozayani A. 2007. The estimation of blood alcohol concentration: Widmark revisited. Forensic Science, Medicine, and Pathology 3: 33-39.

Rashad I, Grossman M, Chou SY. 2006. The super size of America: an economic estimation of body mass index and obesity in adults. Eastern Economic Journal 32: 133-148.

Rice T, Zhu M. 2013. Driver obesity and the risk of fatal injury during traffic collisions. Emergency Medicine Journal forthcoming.

Simmons WO, Zlatoper TJ. 2010. Obesity and motor vehicle deaths in the USA: a state-level analysis. Journal of Economic Studies 37: 544-556.

Sivak M, Schoettle B, Rupp J. 2010. Survival in fatal road crashes: body mass index, gender, and safety belt use. Traffic Injury Prevention 11: 66-68.

Wagenaar AC, Salois MJ, Komro KA. 2009. Effects of beverage alcohol price and tax levels on drinking: a meta-analysis of 1003 estimates from 112 studies. Addiction 104: 1360-0443.

Widmark EMP. 1932. Die theoretischen Grundlagen und the praktische Verwendbarkeit der gerichtlich-medizinischen Alkoholbestimmung. Urban \& Schwarzenberg: Berlin.

Zhao Z, Kaestner R. 2010. Effects of urban sprawl on obesity. Journal of Health Economics 29: 779-787. 